\title{
Study on the Rheological Properties of Ultra-High Temperature CGA Drilling Fluids
}

\author{
Wenxi ZHU ${ }^{1}$ and Xiuhua $\mathrm{ZHENG}^{2}$ \\ School of Engineering and Technology, China University of Geosciences (Beijing), \\ Beijing 100083, China
}

\begin{abstract}
Colloidal gas aphron (CGA) drilling fluids are a kind of environmentallyfriendly underbalanced drilling technique, which has attracted more attention in depleted reservoirs and other low-pressure areas. With the shortage of global oil/gas resources, drilling has gradually shifted to high-temperature and deep wells. Hence, a study on the ultra-high temperature rheology properties of CGA fluids is lacking and urgently needed. In this study, a novel CGA drilling fluid system was prepared by modified starch and amino acid surfactant, and rheological properties after 120$300^{\circ} \mathrm{C}$ aged was investigate. Results show that: (1) Herschel-Bulkley model is the preferred model to predict CGA drilling fluid at ultra-high temperatures; (2) It was proved that CGA drilling fluid is a high-quality drilling fluid with extremely high value of LSRV and shear thinning property within $280{ }^{\circ} \mathrm{C}$. Compared to the traditional XG-based CGA drilling fluid, the improvement of LSRV at ultra-high temperatures is a significant advantage of EST-based CGA drilling fluid which is conducive to carrying cuttings and sealing formation pores.
\end{abstract}

Keywords. Colloidal gas aphron (CGA), drilling fluid system, rheological properties

\section{Introduction}

Colloidal gas aphron (CGA) drilling fluids as a kind of environmentally-friendly underbalanced drilling technique is finding increasing application in depleted reservoirs and other low-pressure areas because of its distinctive characteristic [1]. Aphrons in CGA fluids are microbubbles with a diameter of $\sim 100 \mu \mathrm{m}$ generated by polymers and surfactants. Aphrons are composed of a gas core and multilaminar structure, which can be used as sealing materials by forming an elastic internal bridge without solids in porous mediums/fractures to hinder fluid invasion. Moreover, aphrons can be cleaned up to the ground easily in the production stage, which can reduce the cost associated with stimulation processes. CGA drilling fluids also presents several advantages such as high cutting carrying capacity and an increase in drilling speed [2-4].

The rheology of drilling fluid is one of the most important properties related to safe drilling and the design of drilling parameters. There have been some reports on the rheology of CGA fluid. Arabloo et al. proposed an improved rheological model including

${ }^{1}$ Corresponding Author, Wenxi ZHU, School of Engineering and Technology, China University of Geosciences (Beijing), Beijing 100083, China; Email: zhuwenxidida@163.com.

${ }^{2}$ Email: xiuhuazh@cugb.edu.cn. 
thermodynamic parameters to describe the rheology of CGA fluids prepared with xanthan gum $(\mathrm{XG})$ at $25-45{ }^{\circ} \mathrm{C}$ [5]. Khamehchi et al. investigated the rheological properties of CGA drilling fluids generated by XG and PAC after aging at $49 / 71 / 93{ }^{\circ} \mathrm{C}$ [6]. Results showed that the increase of aging temperature increased the consistency index and decreased the flow behavior index. Xie et al. stated that the apparent viscosity (AV) and yield point of the CGA drilling fluid produced by XG and carboxymethyl cellulose (CMC) decreased to a certain extent before and after aging at $135^{\circ} \mathrm{C}$, which meets the requirements of the site [7].

So far, the research on the rheological properties of CGA is mainly concentrated within $150{ }^{\circ} \mathrm{C}$. With the shortage of global oil/gas resources, drilling has gradually shifted to high-temperature deep reservoirs. For oil and gas reservoirs, the maximum temperature can reach $240{ }^{\circ} \mathrm{C}$, and for high-temperature geothermal wells, it can reach $300^{\circ} \mathrm{C}$ or even higher. Therefore, a study on the ultra-high temperature rheology properties of CGA fluids is urgently needed. In this study, a novel CGA drilling fluid system prepared by modified starch and amino acid surfactant was exposed for the first time, and the rheological properties of the system after aging at ultra-high temperatures $\left(120-300{ }^{\circ} \mathrm{C}\right)$ was systematically discussed.

\section{Materials and Methodology}

The stabilizer used in this paper was modified starch EST synthesized by inverse emulsion polymerization, which can be found in Ref. [8]. The foaming agent was lauryl glycine surfactant $\mathrm{RN}-12$. Anhydrous sodium carbonate $\left(\mathrm{Na}_{2} \mathrm{CO}_{3}\right)$ and bentonite were commercially reagents supplied by Xilong Chemical Reagent Co., Ltd and Weifang Boda Bentonite Co., LTD, respectively.

\subsection{Preparation of CGA Drilling Fluids}

The base mud was prepared by mixing $3 \mathrm{wt} \%$ bentonite and $0.2 \% \mathrm{Na}_{2} \mathrm{CO}_{3}$. Take out 250 $\mathrm{mL}$ base mud, add 2\% EST and stir at 8,000 rpm for $20 \mathrm{~min}$ by using a high-speed mixer (Model WT-2000C). Then, add 3\% RN-12 and stirring at 10,000 rpm for $5 \mathrm{~min}$ to generate aphrons. The high-temperature treated CGA drilling fluid was obtained by putting the prepared fluid into the rolling oven (Model XGRL-4A) at appointed temperatures $\left(120-300^{\circ} \mathrm{C}\right)$ and aged for 16 hours.

\subsection{Rheological Properties}

Rheological properties of the prepared ultra-high temperature fluids were carried out by using Brookfield DV-2 viscometer and six-speed viscometer (Model ZNN-D6). The former was used for measuring the low shear rate viscosity (LSRV) at $0.3 \mathrm{rpm}$. The relationship between shear rate $(\gamma)$ and shear stress $(\tau)$ was recorded by the latter, which was further fitted by four mathematical models: Bingham model, Casson model, Power Law model, and Herschel-Bulkley model, see equations (1)-(4) [9].

$$
\begin{aligned}
& \text { Bingham Model: } \tau=\tau_{0}+\mu_{\mathrm{p}} \gamma \\
& \text { Casson model: } \tau^{0.5}=\tau_{0}+\mathrm{K} \gamma^{0.5}
\end{aligned}
$$




$$
\text { Power Law model: } \tau=\mathrm{K} \gamma^{\mathrm{n}}
$$

$$
\text { Herschel-Bulkley model: } \tau=\tau_{0}+\mathrm{K} \gamma^{\mathrm{n}}
$$

where $\tau_{0}$ is yield point, $\mu_{\mathrm{p}}$ is plastic viscosity, $\mathrm{n}$ is flow behavior index and $\mathrm{K}$ is consistency index. Parameters constrains: $\tau_{0} \geq 0 ; 0<\mathrm{n}<1$; $\mathrm{K}>0$.

\section{Results and Discussion}

\subsection{Optimization of Rheological Models}

As described in section 2.2, figure 1 shows the fitting curves of the four rheological models of the ultra-high temperature aged CGA fluids. The accuracy of fitting was compared by the goodness of fit $\left(\mathrm{R}^{2}\right)$ and root mean square error (RMSE), see figure 2 . The closer the value of $\mathrm{R}^{2}$ is to 1 , the smaller the RMSE value, the higher accuracy is [10].
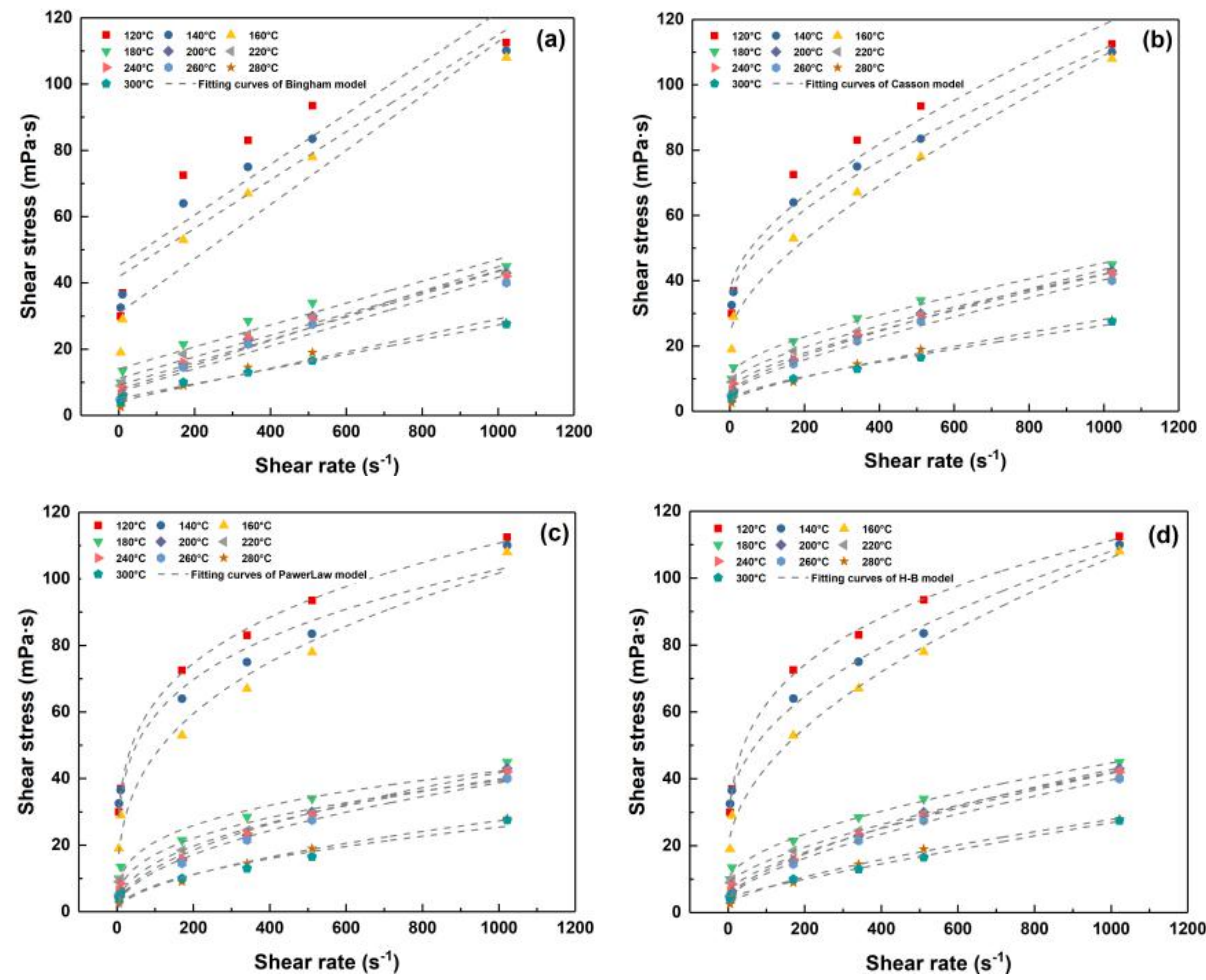

Figure 1. Fitting curves of CGA drilling fluids at appointed aging temperatures: (a) Bingham model; (b) Casson model; (c) Power-Law model; (d) Herschel-Bulkley model.

Results show that the $\mathrm{R}^{2}$ value of four models at different temperatures was in the range of 0.833-0.992(Bingham model), 0.938-0.998(Power-law model), 0.9460.999(Casson model), and 0.994-0.999(Herschel-Bulkley model), respectively. The 
average RMSE value of the Bingham model, Power-law model, Casson model, and Herschel-Bulkley model were 4.15, 2.26, 1.83, and 0.84, respectively. In general, the accuracy of the four rheology models is as follows: Herschel-Bulkley model $>$ Casson model $>$ Power law model $>$ Bingham model. The goodness of fit values of the HerschelBulkley model at different temperatures was all greater than 0.99 , and had the lowest average RMSE value. Hence, it's proved that the Herschel-Bulkley model was the preferred model to describe the rheology behavior of ultra-high temperature CGA fluids, and the rheological parameters obtained were credible.
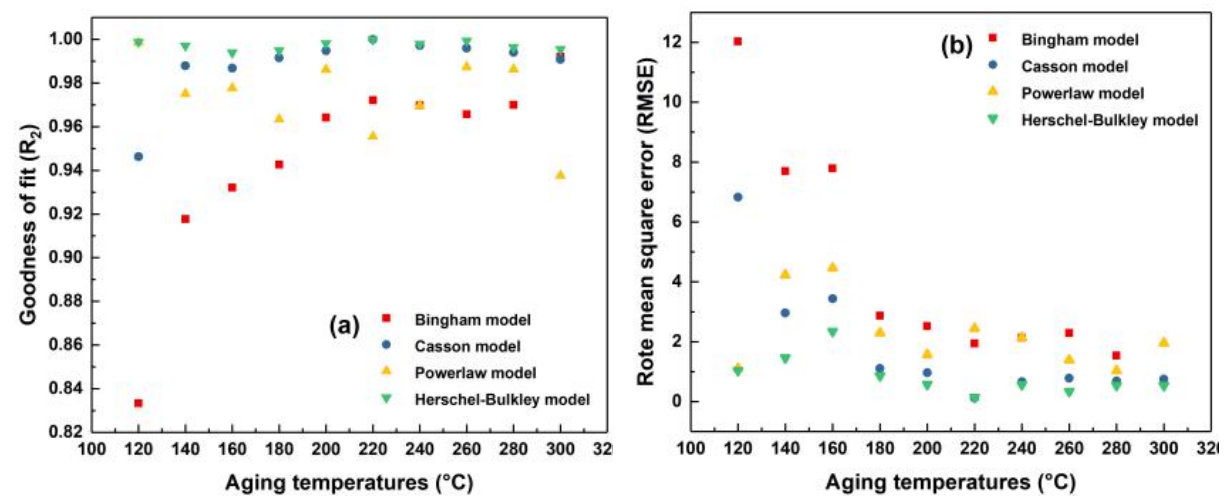

Figure 2. $\mathrm{R}^{2}$ (a) and RMSE (b) of four rheology models when fitting ultra-high temperature CGA fluids.

\subsection{Low Shear Rate Viscosity (LSRV)}

At a low shear rate, the higher the fluid viscosity is, the more conducive to the reverse discharge of cuttings and the bridging and sealing of microbubbles in porous media. The LSRV values of prepared CGA drilling fluids aged at appointed temperatures are as follows: $234000 \mathrm{cp}\left(120^{\circ} \mathrm{C}\right), 136000 \mathrm{cp}\left(140^{\circ} \mathrm{C}\right), 108000 \mathrm{cp}\left(160^{\circ} \mathrm{C}\right), 85582 \mathrm{cp}\left(180^{\circ} \mathrm{C}\right)$, $53189 \mathrm{cp}\left(200^{\circ} \mathrm{C}\right), 38392 \mathrm{cp}\left(220^{\circ} \mathrm{C}\right), 29194 \mathrm{cp}\left(240^{\circ} \mathrm{C}\right), 28394 \mathrm{cp}\left(260^{\circ} \mathrm{C}\right), 23595 \mathrm{cp}$ $\left(280^{\circ} \mathrm{C}\right), 23995 \mathrm{cp}\left(300^{\circ} \mathrm{C}\right)$. The LSRV values gradually decreased with the increase of aging temperatures. It's worth noting that compared with the traditional CGA drilling fluids prepared by xanthan gum (XG) and sodium dodecyl sulfatethe (SDS), the LSRV of the novel CGA drilling fluid system prepared by modified starch and amino acid surfactant was significantly improved. As reported, the LSRV of XG-based CGA fluids was $11198 \mathrm{cP}\left(120^{\circ} \mathrm{C}\right)$ and $5999\left(180{ }^{\circ} \mathrm{C}\right)$, which decreased by $95.2 \%$ and $93.0 \%$ compared with the EST-based CGA drilling fluids [9].

\subsection{Shear Thinning Properties}

Shear-thinning refers to the characteristic that the fluid viscosity decreases with the increase of speed, which is expressed by the flow behavior index (n). The lower the value of $n$, the stronger the shear thinning of the fluid. To effectively open the pump to break rock at the high shear rate and carry cuttings at the low shear rate, high-quality drilling fluid must require a reasonable value of $n(n \leq 0.7)$.

Figure 3 shows the viscosity-rotational speed curves of CGA fluids after aging at 120-300 ${ }^{\circ} \mathrm{C}$. CGA drilling fluids had a high value of viscosity at a low shear rate as described in 3.2. A rapid decrease in viscosity was observed as the rotational speed 
increased from $0.3 \mathrm{rpm}$ to $1 \mathrm{rpm}$. Then the fluid viscosity continued to decrease slightly as the rotational speed gradually increased. Figure 3 indicated that EST-based CGA drilling fluids prepared had a significant shear thinning characteristic at ultra-high temperatures.

According to the discussion in section 3.1, the Herschel-Bulkley model has the highest accuracy in fitting ultrahigh temperature CGA fluids. Therefore, the rheological parameters of the Herschel-Bulkley model as listed in table 1 are highly reliable. Results showed that the $\mathrm{n}$ value increased gradually with the increasing aging temperatures. It means that the flow state of drilling fluid changed from flat laminar flow to peak turbulent flow, and the shear thinning behavior weakened. Within $280^{\circ} \mathrm{C}$, the $\mathrm{n}$ value of CGA fluid ranged from 0.267 to 0.691 , which did not exceed the reasonable range of $n$. The $n$ value of CGA fluid increased to 0.891 at $300{ }^{\circ} \mathrm{C}$, which was beyond the reasonable range. Therefore, a high-temperature resistant flow pattern regulator is suggested to be added to the formulation.

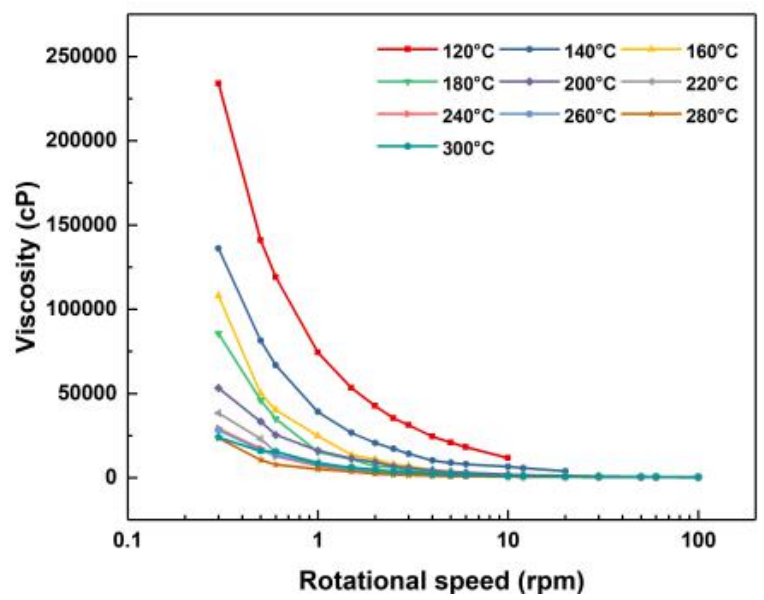

Figure 3. Rotational speed-viscosity curves of CGA drilling fluids under different aging temperatures.

Table 1. Rheological parameters of Herschel-Bulkley model.

\begin{tabular}{lllllllllll}
\hline $\mathbf{T}\left({ }^{\circ} \mathbf{C}\right)$ & $\mathbf{1 2 0}$ & $\mathbf{1 4 0}$ & $\mathbf{1 6 0}$ & $\mathbf{1 8 0}$ & $\mathbf{2 0 0}$ & $\mathbf{2 2 0}$ & $\mathbf{2 4 0}$ & $\mathbf{2 6 0}$ & $\mathbf{2 8 0}$ & $\mathbf{3 0 0}$ \\
\hline$\tau_{0}$ & 4.8 & 26.7 & 17.2 & 9.5 & 4.0 & 8.3 & 6.1 & 3.7 & 2.2 & 4.5 \\
$\mathrm{n}$ & 0.267 & 0.474 & 0.529 & 0.574 & 0.653 & 0.678 & 0.679 & 0.655 & 0.691 & 0.876 \\
$\mathrm{~K}$ & 16.88 & 3.08 & 2.30 & 0.67 & 0.43 & 0.31 & 0.33 & 0.39 & 0.22 & 0.05 \\
\hline
\end{tabular}

\section{Conclusion}

In this paper, a new type of EST-based CGA drilling fluid system composed of modified starch and amino acid surfactant is revealed. The rheological properties at ultra-high temperature $\left(120-300^{\circ} \mathrm{C}\right)$ were investigated and the results showed that: (1) HerschelBulkley model is the preferred model to describe CGA fluid at ultra-high temperatures, and the goodness of fit is up to 0.99 ; (2) Compared to the traditional XG-based CGA drilling fluid, the improvement of LSRV at ultra-high temperatures is a significant 
advantage of EST-based CGA drilling fluid; (3) Within $280^{\circ} \mathrm{C}$, EST-based CGA drilling fluid has been proved to be a high-quality drilling fluid with shear thinning behavior.

\section{Acknowledgments}

This work was supported by the National Natural Science Foundation of China (Grant No. 41872184) of "Properties of Colloid Gas Aphron Drilling Fluid and Its Mechanism of Loss and Formation Protection for High Temperature Geothermal Reservoir".

\section{References}

[1] Growcock F B, Belkin A, Fosdick M, Irving M, O’Connor B and Brookey T 2007 SPE Drilling \& Completion 22 74-80.

[2] Tabzar A, Arabloo M and Ghazanfari M H 2015 J.Nat.Gas.Sc.Eng. 26 895-906.

[3] Ziaee H, Arabloo M, Ghazanfari M H and Rashtchian D 2015 Chem. Eng Res. Des. 93 21-29.

[4] Pasdar M, Kazemzadeh E, Kamari E, Ghazanfari M H and Soleymani M 2018 Colloids and Surfaces aPhysicochemical and Engineering Aspects 537 250-58.

[5] Arabloo M and Shahri M P 2014 J.Pet.Sci.Eng 113 8-22.

[6] Khamehchi E, Tabibzadeh S and Alizadeh A 2016 Petroleum Exploration and Development 43 1076-81.

[7] Xie J Y, Zhou Y X, Geng X H, Lu G L, Liu G C and Hao J H 2014 Advances In Fine Prtrochemicals 15 30-34.

[8] Zhu W X and Zheng X H. 2020 Drilling Fluid \& Completion Fluid 1-11.

[9] Zhu W X, Zheng X H and Li G M 2020 J.Pet.Sci.Eng 186.

[10] Hassani A H and Ghazanfari M H 2017 J.Nat.Gas.Sc.Eng. 42 1-12. 